\title{
A direct comparison of ecological theories for predicting the relationship between plant traits and growth
}

\section{Ellie Goud ( $\nabla$ egoud@uwaterloo.ca )}

University of Waterloo https://orcid.org/0000-0001-5494-5884

\section{Anurag Agrawal}

Cornell University https://orcid.org/0000-0003-0095-1220

Jed Sparks

Cornell University

\section{Article}

Keywords: biomass, CSR, ecophysiology, growth analysis, leaf area, leaf economics spectrum, plant ecology, plant strategies

Posted Date: May 25th, 2021

DOI: https://doi.org/10.21203/rs.3.rs-518839/v1

License: (c) (i) This work is licensed under a Creative Commons Attribution 4.0 International License. Read Full License 
Title

2 A direct comparison of ecological theories for predicting the relationship between plant traits and 3 growth

Authors

Ellie M. Goud*1,2, Anurag A. Agrawal ${ }^{1}$, and Jed P. Sparks ${ }^{1}$

${ }^{1}$ Department of Ecology and Evolutionary Biology, Cornell University, Ithaca, NY 14850, USA

${ }^{2}$ Department of Geography and Environmental Management, University of Waterloo, Waterloo, ON N2L 3G1, Canada

$11 *$ Corresponding author: egoud@ uwaterloo.ca. Current address: Department of Geography and 12 Environmental Management, 200 University Avenue, Waterloo, ON N2L 3G1, Canada.

Keywords: biomass, CSR, ecophysiology, growth analysis, leaf area, leaf economics spectrum, plant ecology, plant strategies

\section{Abstract}

Despite long-standing theory for classifying plant ecological strategies, limited data directly links organismal traits to whole-plant growth. We compared trait-growth relationships based on three prominent theories: growth analysis, Grime's CSR triangle, and the leaf economics spectrum (LES). Under these schemes, growth is hypothesized to be predicted by traits related to biomass investments, leaf structure or gas exchange, respectively. In phylogenetic analyses of 30 diverse milkweeds (Asclepias spp.) and 21 morphological and ecophysiological traits, growth rate varied 50-fold and was best predicted by growth analysis and CSR traits, as well as total leaf area and plant height. Despite two LES traits correlating with growth, they contradicted predictions and leaf traits did not scale with root and stem characteristics. Thus, although combining leaf traits and whole-plant allocation best predicts growth, when destructive measures are not feasible, we suggest total leaf area and plant height, or easy-to-measure traits associated with the CSR

30 classification. 
Predicting variation in plant growth is a long-standing problem in ecology. Because plants largely determine ecosystem productivity, estimating current and future plant growth is increasingly relevant as global change drivers impact ecosystem services ${ }^{1,2}$. As it is typically impractical to measure the total vegetative biomass of a community or ecosystem, an emerging method is to apply plant traits to predict growth rate. These trait-based approaches take advantage of a large body of literature that analyzes co-variation and trade-offs among plant traits ${ }^{3-6}$. Given that morphological and physiological characters are central to resource acquisition and allocation, they are likely to shape plant productivity in predictable ways.

Three classic approaches have attempted to distill plant diversity into cohesive strategies and estimate growth based on defining characteristics: growth analysis, Grime's CSR triangle, and the leaf economics spectrum (Table 1). In growth analysis, growth rate is predicted by the relative allocation of biomass among roots, stems, and leaves ${ }^{3,7}$. Faster growing plants are expected to invest more in leaves relative to stems and roots. Due to the importance of leaf investment, growth rates are additionally dependent on specific leaf area (SLA), the ratio of leaf area to dry mass. Grime's CSR (competition-stress tolerant-ruderal) framework predicts that these three plant strategies have repeatedly evolved in response to combinations of stress and disturbance ${ }^{8}$. Until recently, the CSR framework was conceptual rather than empirically traitbased. However, Pierce et al. 2016 showed that three leaf traits were predictive of the scheme: average leaf surface area (individual leaf size, LS), SLA, and leaf dry matter content (LD). In this context, the C-strategy is defined by large LS and intermediate LD and SLA. The S-strategy has small LS and SLA with large LD, and R-strategy has small LS, small LD and large SLA 9 .

The most commonly applied trait-based framework is the leaf economics spectrum, which describes patterns of co-variation among six leaf traits: leaf lifespan, SLA, leaf nitrogen (N) and phosphorus $(\mathrm{P})$ contents, rates of light-saturated photosynthesis $\left(\mathrm{A}_{\max }\right)$ and dark respiration $\left(\mathrm{R}_{\mathrm{d}}\right)$. Together, these traits represent the return on investment of fixed carbon at the leaf-level ${ }^{4,10}$. On one end of the spectrum is a 'resource-acquisitive' strategy with short leaf lifespan and larger SLA, N, P, $A_{\max }$, and $\mathrm{R}_{\mathrm{d}}$. On the other end is a 'resource-conservative' strategy with the opposite combination of trait values. It is generally predicted that plants with resource-acquisitive leaf traits have relatively faster growth rates than those with resource-conservative leaves ${ }^{10}$. As such, leaf economics traits have been applied to predict growth at genotypic, community, ecosystem, and global scales, often using a subset of traits as direct proxies for plant growth ${ }^{11,12}$.

Despite the intuitive appeal of these theories, experiments that directly use traits to predict whole-plant growth are surprisingly limited. Studies to date show mixed results, including significant ${ }^{13}$ and non-significant ${ }^{14}$ trait-growth rate relationships. Inconsistent results could be influenced by environmental variation ${ }^{15,16}$, species interactions ${ }^{13}$, or because studies compare plants at different scales - from broadly divergent taxonomic and functional groups at the community scale, to closely related plants within a clade, to among plant individuals within a species ${ }^{17,18}$.

In an effort to link these theoretical frameworks to observed growth rate, we simultaneously tested these growth-trait predictions (Table 1) using a diverse set of closely related species in a multivariate phylogenetic analysis. We measured 21 traits: leaf, stem and root biomass fractions (LMF, SMF, RMF) and SLA for growth analysis, CSR-associated traits (LS, LD, SLA), and leaf 
economic spectrum traits $\left(A_{\max }, R_{d}, N, P, S L A\right)$. We included six traits that we expected to be predictors of plant growth but are not used in any of the three approaches: total leaf area (LA), leaf thickness ( $\left.\mathrm{L}_{\mathrm{th}}\right)$, leaf carbon isotope ratio $\left(\delta^{13} \mathrm{C}\right)$, specific root length (SRL), root clonality, seed mass (SM), and plant height $(\mathrm{H})$. Growing species from the same genus under controlled, common resource conditions in a growth chamber allowed us to compare growth rates and traits of interest within a similar functional group (i.e., all $\mathrm{C}_{3}$, diploid, herbaceous, and perennial) that share a recent common ancestor, and yet display large variation in growth, habitat affinities, and traits.

\section{Materials and methods}

Study system and plant growth conditions

We assessed growth-trait relationships across 30 closely related yet functionally diverse milkweed species. Milkweeds (Asclepias spp., Apocynaceae) are herbaceous perennials that display remarkable variation in morphology, ranging from desert subshrubs with small, narrow leaves (e.g., A. linaria, A. subulata) to large, highly productive plants of temperate and subtropical wetlands with large, broad leaves (e.g., A. curassavica, A. incarnata) ${ }^{19}$. Milkweeds vary widely in growth rate and traits when grown under common garden conditions ${ }^{14,20}$. Seeds collected by colleagues or purchased from native plant suppliers were germinated by moistening and stratifying at $4^{\circ} \mathrm{C}$ for at least 10 days and then at $28^{\circ} \mathrm{C}$ for 3 days. Seedlings were planted in $500 \mathrm{ml}$ plastic pots and grown in Metro Mix soil (Scotts-Sierra, Marysville, OH, USA) in a walk-in growth chamber (Conviron CMP 6050) for 6 weeks at $26^{\circ} \mathrm{C}$ during a 14-hour day and $24^{\circ} \mathrm{C}$ during a 10 -hour night with an average relative humidity of $50 \%$.

\section{Trait and biomass measurements}

103 We sampled five replicate plants per species for trait and growth measurements. Seed mass (SM) was calculated before germination by dividing the total seed mass $(\mathrm{g})$ by the number of seeds used for germination ( $\mathrm{n}=30$ per species). We measured leaf gas exchange rates using a LI-COR LI-6400 $\mathrm{CO}_{2}$ gas exchange analyzer (LI-COR, Lincoln, NE) on one fully expanded leaf per plant at 36 - 41 days old. We generated light response curves to obtain the light intensity (photosynthetically active radiation, PAR, spanning $0-2000 \mu \mathrm{mol}$ photons $\mathrm{m}^{-2} \mathrm{~s}^{-1}$ ) at which photosynthesis saturated $\left(A_{\max }\right)$. Leaf dark respiration $\left(R_{d}\right)$ was measured at a PAR of zero. Leaf humidity inside the cuvette was maintained between $45-60 \%$, and the block temperature was maintained at $25^{\circ} \mathrm{C}$.

At the time of harvest, the height of each plant was measured $(\mathrm{H}, \mathrm{cm})$, total leaf number was recorded, leaves were removed from the stems, and roots were separated and washed to remove soil. For each individual, total leaf area (LA) was measured using a LI-COR LI-3100 leaf-area meter (LI-COR, Lincoln, NE). Specific root length (SRL) was measured as the ratio of root length $(\mathrm{cm})$ to dry mass using subsamples of lateral roots $(\mathrm{n}=10$ per plant). Root clonality was measured as the number of buds on roots, rhizomes and caudices of each plant ${ }^{21}$. After fresh weight measurements of leaves, stems, and roots, samples were oven-dried at $60^{\circ} \mathrm{C}$ for 48 hours and measured for dry mass (g). Average leaf area (leaf size, LS) was calculated by dividing LA by the total number of leaves. Specific leaf area (SLA) was measured as LA divided by total oven-dried leaf mass (g). Leaf density (LD) was measured as oven-dry leaf mass (mg), divided by water-saturated fresh mass $(\mathrm{g})$. Leaf thickness $\left(\mathrm{L}_{\mathrm{th}}\right)$ was estimated as SLA * LD ${ }^{22}$. 
Carbon isotope ratios and $\mathrm{N}$ percent element of leaf material was measured using a continuous flow isotope ratio mass spectrometer (Thermo Scientific Delta V Advantage) coupled to an elemental analyzer (Carlo Erba NC2500). Isotope ratios are expressed as $\delta$ values (per mil):

$$
\delta^{13} \mathrm{C}=\left(\mathrm{R}_{\text {sample }} / \mathrm{R}_{\text {standard }}-1\right) \times 1000(\%)
$$

where $\mathrm{R}_{\text {sample }}$ and $\mathrm{R}_{\text {standard }}$ are the ratios of heavy isotope to light isotope of the sample relative to Vienna-Pee-Dee Belemnite, the international standard for C. Mass spectrometry and elemental analyses were performed at the Cornell University Stable Isotope Laboratory. Leaf P content was measured by dry ash analysis at the Cornell Nutrient Analysis Laboratory. Leaf N and P were calculated on both a leaf dry mass and leaf area basis.

Plant growth can be defined and measured in various ways, including relative growth (RGR) and absolute growth rate (GR, the total oven-dried biomass accrued over the number of days from seedling emergence until the end of the experiment in $\left.\mathrm{g} \mathrm{d}^{-1}\right)$. RGR requires multiple harvests and can often constrain replication ${ }^{7}$. We found a robust correlation between RGR and GR $\left(R^{2}=\right.$ $0.97, \mathrm{p}<000.1)$ for four milkweed species that represented the range of functional variation across the 30 species: A. curassavica, A. incarnata, A. pumila, A. verticillata. Therefore, we used GR for the purpose of this broader study.

\section{Statistical analyses}

We assessed the relative ability of each trait to predict growth using generalized linear models (GLM), and tested for potential effects of shared evolutionary history using phylogenetic generalized least squares (PGLS) regressions using the 'pgls' function of the caper package in $\mathrm{R}$ 23. A maximum likelihood phylogeny of 111 Asclepias species was pruned with the retention of branch lengths to create a phylogram for the 30 species (Figure S1). Briefly, the phylogeny was estimated from three non-coding plastid genome regions: $r p l 16$ intron, $\operatorname{trn} C^{G C A}-r p o B$ intergenic spacer, and the contiguous $\operatorname{trn} S^{G C U}-\operatorname{trn} G^{U U C}$ intergenic spacer/trn $G^{U U C}$ intron ${ }^{24}$. We estimated the phylogenetic signal of growth rate and all traits by calculating Pagel's $\lambda$ using the 'phylosig' function in the picante R-package ${ }^{25}$. We included the standard error of the mean for each variable. A $\lambda$-value of 1 indicates that trait similarity among species is directly proportional to the extent of shared evolutionary history, while a $\lambda$-value $=0$ indicates no relationship between shared ancestry and trait values. For the PGLS analyses, linear regression parameters were estimated from a $\lambda=1$ model.

We used Ward's agglomerative clustering and principal components analysis using the 'vegan' R-package ${ }^{26}$ to classify the 30 species into distinct groups, hereafter referred to as 'strategies'. We grouped species based on (1) growth analysis traits, (2) CSR traits, (3) LES traits, and (4) all 21 measured traits. We then used Kendall's coefficient of concordance (999 permutations) to identify how many strategies identified by Ward's clustering were significantly distinct and which species were significantly contributing to each strategy ${ }^{27}$. We also classified species using the CSR method outlined in Pierce et al. 2016, where ratios of C:S:R values were calculated based on LS, LD, and SLA for each species. We used linear regression to assess relationships between GR and the first two principal components axes for each strategy scheme. Linear 
regression models were directly compared by Akaike information criterion (AIC). All analyses were performed in R3.5.3 ${ }^{28}$.

\section{Results}

\section{Growth-trait relationships across species}

Across 30 milkweed species, individual plant growth ranged over 50 -fold, from $0.005 \mathrm{~g} \mathrm{~d}^{-1}$ to $0.258 \mathrm{~g} \mathrm{~d}^{-1}$ (Table S1). Overall, in phylogenetically controlled analyses (PGLS), three traits each explained over $50 \%$ of the variation in growth among the 30 Asclepias species (total leaf area, nitrogen content on a mass basis, and phosphorus content on an area basis). In total, growth rate positively correlated with $\delta^{13} \mathrm{C}, \mathrm{H}, \mathrm{LA}, \mathrm{LD}, \mathrm{LMF}, \mathrm{LS}$, area-based photosynthetic rate (Aarea) and $\mathrm{SMF}$ and negatively correlated with leaf $\mathrm{N}$ and $\mathrm{P}$ (analyses and abbreviations in Table 2). Growth did not correlate with $\mathrm{R}_{\mathrm{d}}$ (per mass or per area), $\mathrm{L}_{\text {th }}$, mass-based photosynthetic rate (Amass), root clonality, RMF, SM, SLA, or SRL. Accounting for shared evolutionary history did not change the relationships, with the exception of LMF (not significant in PGLS) and Amass (became significant in PGLS). Nine of the 21 traits showed phylogenetic signal ( $\lambda$-value $>0.70$, $P<0.05): \delta^{13} \mathrm{C}, \mathrm{H}, \mathrm{R}_{\text {mass, }} \mathrm{LD}, \mathrm{LMF}, \mathrm{N}_{\text {mass, }}$ Lth, SM, and SMF (Table 2).

\section{Plant strategies}

In order to assess growth-trait relationships at the level of plant strategies, we classified species into groups using diagnostic traits of the three theories (Table 1, 3). Using growth analysis traits, we identified three strategies primarily defined by the relative allocation between above and belowground biomass (PC1 =70\%; Fig. 1A). When grouped by CSR traits, species distributed among four strategies corresponding to CR, CSR, SR and S. Consistent with previous studies ${ }^{9,29}$, variation in LS was orthogonal to variation in SLA and LD, with differences among groups driven primarily by variation in average leaf size (LS) (PC1 = 93\%; Fig. 1D). For LES traits, species distributed among three strategies primarily defined by variation in $\mathrm{A}_{\text {mass }}, \mathrm{A}_{\text {area }}, \mathrm{P}_{\text {area, }}$, and $\mathrm{N}_{\text {area }}(\mathrm{PC} 1=51 \%)$, and secondarily by leaf $\mathrm{N}_{\text {mass }}$ and $\mathrm{Pmass}(\mathrm{PC} 2=30 \%$; Fig. $1 \mathrm{G})$.

In addition to these well-established schemes, we classified species using all 21 traits measured in this study. Using this approach, the 30 species distributed among four groups (Fig. 1J). Consistent with global trait analyses ${ }^{5}$, the first PC axis (52\%) corresponded to plant size-related traits LA, H, LS, biomass fractions (LMF, SMF, RMF) and LD. The second PC axis (19\%) corresponded to leaf economic and metabolic traits $\left(\delta^{13} \mathrm{C}, \mathrm{N}, \mathrm{P}, \mathrm{A}_{\max }, \mathrm{L}_{\mathrm{th}}\right)$. We describe the four strategies identified from all measured traits by their overall plant size (small, medium, large) and primary biomass allocation (roots, leaves, stems).

\section{Growth-trait relationships across plant classification schemes}

Across strategies, growth correlated most strongly with the first principal component axis (PC1) of growth analysis $\left(R^{2}=0.91, P<0.0001\right.$, AIC $=-90$; Fig. 1B), and for all 21 traits combined $\left(R^{2}\right.$ $=0.84, P<0.0001$, AIC $=-72$; Fig. $1 \mathrm{~K})$, driven primarily by variation in LA and H (Table S2). Growth also positively correlated with PC1 of CSR $\left(R^{2}=0.49, P<0.0001\right.$, AIC = -39; Fig. 1E). Growth negatively correlated with PC1 of LES $\left(R^{2}=0.29, P=0.001\right.$, AIC $\left.=-29\right)$, PC2 of LES $\left(R^{2}=0.31, P=0.001\right.$, AIC $\left.=-30\right)$, and PC2 of all measured traits $\left(R^{2}=0.16, P=0.006\right.$, AIC $=-$ 26). Growth was not predicted by PC2 of GA or CSR (both $R^{2}<1.0, P>0.05,-20<\mathrm{AIC}<-19$; Fig. 1C,F). 


\section{Discussion}

Here we bridge classic ecological theory with current trait-based approaches in order to better predict whole-plant growth from functional traits. We tested predictions that faster growing plant species will (1) allocate more biomass to aboveground tissues (based on growth analysis); (2) have larger LS, SLA and lower LD (based on CSR); or (3) have 'acquisitive' leaf traits defined by larger $A_{\max }, R_{d}, N, P$, and SLA (based on LES) relative to slower growing plants. The results supported predictions of growth analysis and CSR, but not LES. Remarkably, although two LES traits did have strong predictive power ( $\mathrm{N}$ per leaf mass and $\mathrm{P}$ per leaf area, contributing to multivariate correlations, Fig. 1), these traits were predictive in the opposite direction to that derived from LES theory such that more $\mathrm{N}$ and $\mathrm{P}$ predicted less growth. Overall, differences in growth rate were driven more by whole-plant (e.g., LA, SMF) than individual leaf-level traits (e.g., SLA), in part because of the contribution of root and stem characteristics that did not scale with individual leaf traits. Combining organ-specific and whole-plant traits provided a more integrated view of plant strategies that was better able to predict growth.

For decades, plant mass fractions have been successfully applied to estimate variation in growth rate, and our study is no exception. Indeed, growth analysis traits together were the strongest predictors of whole-plant growth across a diverse set of 30 milkweed species (Fig. 1B). Although theoretically and empirically appealing, the major shortcoming with growth analysis is feasibility - it is typically not practical to obtain the necessary root mass fraction that is critical for growth analysis. Alternatively, the CSR triangle has been a classic predictor of plant strategic variation for decades, but quantitative metrics were not developed until recently ${ }^{9}$. Recent studies show promising applications of CSR defined by leaf traits to better describe functional diversity ${ }^{30}$ and species interactions ${ }^{31}$ within ${ }^{32,33}$ and across ${ }^{9,30,31}$ species. In this novel application of CSR to predict plant growth, we found strong growth-trait relationships across species, driven primarily by variation in average leaf size with minimal contributions from LD and SLA (Fig. 1 F, J).

Variation in total leaf area (LA) was by far the strongest and most consistent predictor of plant growth across all species individually $\left(R^{2}=0.6\right.$; Table 2$)$ and in multivariate plant strategies (associated with PC1; Fig. 1F, H; Table S2). When all 21 traits were considered together, plant height was the second-best predictor of growth (associated with PC1; Fig. 1F, H; Table S2). Specifically, two strategies with similar growth and LA differed in height, suggesting that LA may be most predictive for plants that invest primarily in photosynthesizing leaf biomass, while plant height may be most effective for plants investing in more structural stem biomass. These results are consistent with positive correlations with ecosystem productivity and LA ${ }^{34,35}$ and height ${ }^{35,36}$. Although total leaf area and height are not considered in growth analysis, LES or CSR theories, height is a key component of plant size and has been used to differentiate among plant functional diversity and ecological strategies at local ${ }^{36}$ and global scales ${ }^{5}$. Importantly, LA and height can be remotely sensed, which is particularly useful where it is not feasible to directly measure traits for all individuals (e.g., forest canopies) or for repeated measurements that require non-destructive sampling ${ }^{35,37}$.

\section{Leaf economics spectrum}


The leaf economics spectrum (LES) has gained considerable attention and is often turned to as the primary approach to understand plant functional diversity ${ }^{5}$. However, the work presented here suggests that LES traits do not predict within-clade differences in plant growth as expected; resource-acquisitive leaf traits negatively correlated with growth and SLA was not predictive of growth at the scales that we investigated. We note that although our multivariate analysis of five out of six LES traits yielded strong predictive power for growth rate (PC1 and PC2 combining to $60 \%$ of the variation, Fig. 1), the fact that these were not in the expected direction and that these traits can be challenging to measure, suggest caution in using the LES framework to predict growth within clades. The best application of LES traits to predict growth may be in comparing broadly divergent plant groups (e.g., angiosperms vs gymnosperms), rather than being generalizable across all scales of biological organization.

Species groups identified by LES traits did not adhere to a linear spectrum of increasing leaf economics or nutrient concentrations with increasing growth. Rather, each multivariate grouping expressed a mixture of 'acquisitive' and 'conservative' trait values. For example, some plants with resource 'conservative' leaves (e.g., small, thick leaves with low $\mathrm{N}$ and $\mathrm{P}$ ) grew considerably faster than species with the opposite set of resource 'acquisitive' traits because these plants were taller and had larger overall LA. Similarly, species with the largest growth had lower foliar $\mathrm{N}$ and $\mathrm{P}$ and similar rates of leaf gas exchange than other, less productive species.

In contrast to predictions, leaf gas exchange $\left(A_{\max }, R_{d}\right)$ were not strong predictors of growth (Fig. 1, Table 2). When all 21 traits were considered, the four plant strategies identified had similar average $A_{\max }$ and $R_{d}$, suggesting that the area available for photosynthesis (i.e., LA, LS) is more important for growth than instantaneous gas exchange rates in this plant group. As an alternative to instantaneous gas exchange rates, we measured leaf carbon isotope composition $\left(\delta^{13} \mathrm{C}\right)$. Contrary to expectations, the fastest growing plants were the most enriched in $\delta^{13} \mathrm{C}$, which is typically associated with slower rates of leaf metabolism ${ }^{14,38}$. For milkweeds, species achieved faster growth by producing numerous large leaves and tall stems. Together, results for instantaneous leaf-level gas exchange rates and $\delta^{13} \mathrm{C}$ suggest that the influence of leaf metabolism is often overwhelmed by differences in total plant LA and, therefore, does not consistently scale to whole-plant growth ${ }^{14,20}$.

Both leaf $\mathrm{N}$ and $\mathrm{P}$ are predicted to positively correlate with growth due to the functional need for $\mathrm{N}$ and $\mathrm{P}$ in photosynthesis ${ }^{4,39}$. However, non-linear or insignificant relationships are frequently reported ${ }^{40,41}$. Here, growth negatively correlated with leaf $\mathrm{N}$ and P (Fig. 1G-I; Table 2), driven by higher $\mathrm{N}$ and $\mathrm{P}$ content yet lower growth in species from arid environments (e.g., $A$. brachystephana, A. mexicana). Higher leaf $\mathrm{N}$ without an accompanying increase in photosynthesis and growth is common for many plants from dry or high irradiance environments that retain leaf $\mathrm{N}$ and $\mathrm{P}^{16,42,43}$ and can operate at a higher leaf $\mathrm{N}$, with the high leaf $\mathrm{N}$ serving to economize water use during photosynthesis ${ }^{44,45}$. Further, not all $\mathrm{N}$ is used for photosynthesis, and recent estimates indicate that herbaceous plants may invest less than $50 \%$ of their leaf $\mathrm{N}$ to photosynthesis, with the remaining $\mathrm{N}$ invested in compounds that support structural and defensive functions ${ }^{46}$. Thus, positive linear relationships between leaf $\mathrm{N}, \mathrm{P}$ and growth are clearly not universal.

\section{Synthesis and speculation}


Although SLA is a component of all three theories, and one of the most commonly reported plant functional traits, growth did not correlate with SLA across species or strategies. SLA represents the investment in leaf area per unit leaf mass, reflecting a potential tradeoff between productivity (i.e., area available for carbon capture), and structural investment (i.e., mass of proteins, carbohydrates, and cell walls). Inconsistent or insignificant correlations between SLA and growth or growth proxies (e.g., leaf gas exchange) are commonly reported ${ }^{40,47,48}$. Thus, although SLA may correlate with growth across broadly divergent species, inconsistencies at smaller scales are likely driven by co-variation with other morphological traits (e.g., LD, Lth) and environmental conditions that are largely independent of growth rate variation ${ }^{17}$.

There is increasing interest in whether functional trait correlations generally apply across scales. Particularly for LES traits, an emerging literature has considered cross-scale relationships. At large taxonomic (e.g., from Arabidopsis to Sequoia) and spatial (e.g., cross-continental ecosystems) scales, patterns of LES trait covariation appear strong and relatively consistent ${ }^{5,6,15}$. However, at finer scales (e.g., within-genus, within-habitat, etc.) patterns are far less consistent and sometimes contrary to theory ${ }^{11,12,49-51}$, as we also found here for milkweeds. This is not necessarily surprising, as trait relationships are often only predictive at the largest scales, where both the range of expression and fundamental biological attributes are revealed ${ }^{6,50-52}$.

In this study, size-related anatomical traits consistently predicted whole-plant growth, while leaf economic and metabolic traits did not. Although we found specific traits to be predictive of growth, we caution against using single traits as their relevance as a consistent predictor of growth is likely to be highly clade-specific. Still, the generality of plant size and CSR-related traits (average leaf size, leaf dry matter content, and SLA) appears the most promising across scales. Practically speaking, these traits can be measured inexpensively and non-destructively, facilitating both use from databases and large-scale field sampling.

The species we studied were distributed along multivariate axes defined by traits, including LES, irrespective of variation in growth rate. In other words, plant size may define growth differences, while economics and metabolism may better differentiate between variation in plant ecology and life-history (Goud et al. 2019). This is consistent with global analyses that found diversity in plant form and function to fall along two major axes of variation related to plant size and leaf economics ${ }^{5}$. Indeed, not all ecological strategies are directly related to growth rate. For example, strategies largely defined by secondary metabolism, such as pollination and defense syndromes, focus on traits that often show little direct connection with grow rate (e.g., flower color, toxic secondary compounds). Therefore, although some traits, and LES traits in particular, may not predict variation in growth as expected, they do capture other important axes of plant functional diversity. Evolutionarily conserved traits within clades are an important driver of differences that promote biodiversity. Ultimately the extent of clade-specificity in which traits or suites of traits predict growth will be highly revealing, not only in terms of scaling generalities, but also in terms of clade-specific traits or strategies that modify expectations. 
We thank Amy Hastings and Patricia Jones for help with seed germination and growth protocols. We are grateful to Kim Sparks, John Pollack, Kelsey Jensen and Michael Roddy for help in the lab and technical assistance, and to Ben Johnson and Fiona Soper for helpful feedback on the manuscript. This work was funded by the Andrew W. Mellon Foundation, the David R. Atkinson Center for a Sustainable Future (Cornell University), and by US National Science Foundation (IOS-1907491). The authors have no conflicts of interest to declare.

Statement of authorship: EMG, AAA, and JPS conceived of the research idea and wrote the

\section{References}

1. Helbig, M. et al. Direct and indirect climate change effects on carbon dioxide fluxes in a thawing boreal forest-wetland landscape. Global Change Biology 23, 3231-3248 (2017).

2. Arndt, K. A. et al. Arctic greening associated with lengthening growing seasons in Northern Alaska. Environ. Res. Lett. 14, 125018 (2019).

3. Lambers, H. \& Poorter, H. in Advances in Ecological Research Volume 23 23, 187-261 (Elsevier, 1992).

4. Wright, I. J. et al. The worldwide leaf economics spectrum. Nature 428, 821-827 (2004).

5. Dìaz, S. et al. The global spectrum of plant form and function. Nature 529, 167-171 (2015).

6. Enquist, B. J. et al. A general integrative model for scaling plant growth, carbon flux, and functional trait spectra. Nature 449, 218-222 (2007).

7. Evans, G. C. The quantitative analysis of plant growth. 1, (Univ of California Press, 1972).

8. Grime, J. P. Evidence for the Existence of Three Primary Strategies in Plants and Its Relevance to Ecological and Evolutionary Theory. The American Naturalist 111, 11691194 (1977).

9. Pierce, S. et al. A global method for calculating plant CSR ecological strategies applied across biomes world-wide. Funct Ecol 31, 444-457 (2016).

10. Reich, P. B. The world-wide 'fast-slow' plant economics spectrum: a traits manifesto. $J$ Ecol 102, 275-301 (2014).

11. Grady, K. C. et al. Conservative leaf economic traits correlate with fast growth of genotypes of a foundation riparian species near the thermal maximum extent of its geographic range. Funct Ecol 27, 428-438 (2013).

12. Blonder, B. et al. Testing models for the leaf economics spectrum with leaf and wholeplant traits in Arabidopsis thaliana. AoB Plants 7, 1-12 (2015).

13. Bongers, F. J. et al. Growth-trait relationships in subtropical forest are stronger at higher diversity. J Ecol 108, 256-266 (2020).

14. Goud, E. M., Sparks, J. P., Fishbein, M. \& Agrawal, A. A. Integrated metabolic strategy: A framework for predicting the evolution of carbon-water tradeoffs within plant clades. $J$ Ecol 107, 1633-1644 (2019).

15. Wright, I. J. et al. Assessing the generality of global leaf trait relationships. New Phytol 166, 485-496 (2005). 
16. Maire, V. et al. Global effects of soil and climate on leaf photosynthetic traits and rates. Global Ecology and Biogeography 24, 706-717 (2015).

17. Edwards, E. J., Chatelet, D. S., Sack, L. \& Donoghue, M. J. Leaf life span and the leaf economic spectrum in the context of whole plant architecture. $J$ Ecol 102, 328-336 (2014).

18. Messier, J., McGill, B. J., Enquist, B. J. \& Lechowicz, M. J. Trait variation and integration across scales: is the leaf economic spectrum present at local scales? Ecography 40, 685697 (2016).

19. Woodson, R. E., Jr. The North American Species of Asclepias L. Annals of the Missouri Botanical Garden 41, 1-211 (1954).

20. Agrawal, A. A. et al. Phylogenetic ecology of leaf surface traits in the milkweeds (Asclepias spp.): chemistry, ecophysiology, and insect behavior. New Phytol 183, 848867 (2009).

21. Pellissier, L. et al. Different rates of defense evolution and niche preferences in clonal and nonclonal milkweeds (Asclepias spp.). New Phytol 209, 1230-1239 (2016).

22. Pérez-Harguindeguy, N. et al. New handbook for standardised measurement of plant functional traits worldwide. Aust. J. Bot. 61, 167-68 (2013).

23. Orme, D. et al. Caper: comparative analyses of phylogenetics and evolution in R. $R$ package version 0.5 2, 458 (2012).

24. Fishbein, M. et al. Phylogenetic Relationships of Asclepias(Apocynaceae) Inferred from Non-coding Chloroplast DNA Sequences. Systematic Botany 36, 1008-1023 (2011).

25. Kembel, S. W. et al. Picante: R tools for integrating phylogenies and ecology. Bioinformatics 26, 1463-1464 (2010).

26. Oksanen, J. et al. vegan: Community Ecology Package, Version 2.5.-6. R Foundation for Statistical Computing (2019).

27. Legendre, P. \& Legendre, L. Numerical ecology. 24, 1-990 (Elsevier, 2012).

28. R Core Team. $R$ : A language and environment for statistical computing. $R$ Foundation for Statistical Computing (2019).

29. Li, Y. \& Shipley, B. An experimental test of CSR theory using a globally calibrated ordination method. PLoS ONE 12, e0175404- (2017).

30. Dayrell, R. L. C. et al. Ontogenetic shifts in plant ecological strategies. Funct Ecol 32, 2730-2741 (2018).

31. Zanzottera, M., Fratte, M. D., Caccianiga, M., Pierce, S. \& Cerabolini, B. E. L. Community-level variation in plant functional traits and ecological strategies shapes habitat structure along succession gradients in alpine environment. Community Ecology 562, 1-11 (2020).

32. Vasseur, F. et al. Climate as a driver of adaptive variations in ecological strategies in Arabidopsis thaliana. Annals of Botany 122, 935-945 (2018).

33. Astuti, G., Ciccarelli, D., Roma-Marzio, F., Trinco, A. \& Peruzzi, L. Narrow endemic species Bellevalia webbianashows significant intraspecific variation in tertiary CSR strategy. Plant Biosystems - An International Journal Dealing with all Aspects of Plant Biology 153, 12-18 (2018).

34. Litton, C. M., Sandquist, D. R. \& Cordell, S. A non-native invasive grass increases soil carbon flux in a Hawaiian tropical dry forest. Global Change Biology 14, 726-739 (2008).

35. Goud, E. M., Moore, T. R. \& Roulet, N. T. Predicting peatland carbon fluxes from nondestructive plant traits. Funct Ecol 31, 1824-1833 (2017). 
36. Westoby, M. A leaf-height-seed (LHS) plant ecology strategy scheme. Plant Soil 199, 213-227 (1998).

37. Davidson, S. J. et al. Linear disturbances shift boreal peatland plant communities toward earlier peak greenness. Earth and Space Science Open Archive 1-20 (2021). doi:10.1002/essoar.10506838.1

38. Ellsworth, P. Z., Ellsworth, P. V. \& Cousins, A. B. Relationship of leaf oxygen and carbon isotopic composition with transpiration efficiency in the $\mathrm{C} 4$ grasses Setaria viridis and Setaria italica. Journal of Experimental Botany 68, 3513-3528 (2017).

39. Walker, A. P. et al. The relationship of leaf photosynthetic traits - Vcmaxand Jmax- to leaf nitrogen, leaf phosphorus, and specific leaf area: a meta-analysis and modeling study. Ecol Evol 4, 3218-3235 (2014).

40. Midgley, G. F., Aranibar, J. N., Mantlana, K. B. \& Macko, S. Photosynthetic and gas exchange characteristics of dominant woody plants on a moisture gradient in an African savanna. Global Change Biology 10, 309-317 (2004).

41. Feng, X. \& Dietze, M. Scale dependence in the effects of leaf ecophysiological traits on photosynthesis: Bayesian parameterization of photosynthesis models. New Phytol 200, 1132-1144 (2013).

42. Field, C., Merino, J. \& Mooney, H. A. Compromises between water-use efficiency and nitrogen-use efficiency in five species of California evergreens. Oecologia 60, 384-389 (1983).

43. Wright, I. J., Reich, P. B. \& Westoby, M. Strategy shifts in leaf physiology, structure and nutrient content between species of high- and low-rainfall and high- and low-nutrient habitats. Funct Ecol 15, 423-434 (2001).

44. Wright, I. J., Reich, P. B. \& Westoby, M. Least-Cost Input Mixtures of Water and Nitrogen for Photosynthesis. The American Naturalist 161, 98-111 (2003).

45. Schrodt, F. et al. Foliar trait contrasts between African forest and savanna trees: genetic versus environmental effects. Functional Plant Biol. 42, 63-83 (2015).

46. Ghimire, B. et al. A global trait-based approach to estimate leaf nitrogen functional allocation from observations. Ecol Appl 27, 1421-1434 (2017).

47. Reich, P. B., Walters, M. B., Ellsworth, D. S. \& Uhl, C. Photosynthesis-nitrogen relations in Amazonian tree species. Oecologia 97, 73-81 (1994).

48. Poorter, H. \& Evans, J. R. Photosynthetic nitrogen-use efficiency of species that differ inherently in specific leaf area. Oecologia 116, 26-37 (1998).

49. Mason, C. M. \& Donovan, L. A. Evolution of the leaf economics spectrum in herbs: Evidence from environmental divergences in leaf physiology across Helianthus(Asteraceae). Evolution 69, 2705-2720 (2015).

50. Agrawal, A. A. A scale-dependent framework for trade-offs, syndromes, and specialization in organismal biology. Ecology 101, 243 (2020).

51. Anderegg, L. D. L. et al. Within-species patterns challenge our understanding of the leaf economics spectrum. Ecology Letters 21, 734-744 (2018).

52. Enquist, B. J., West, G. B., Charnov, E. L. \& Brown, J. H. Allometric scaling of production and life-history variation in vascular plants. Nature 401, 907-911 (1999). 
A Growth analysis

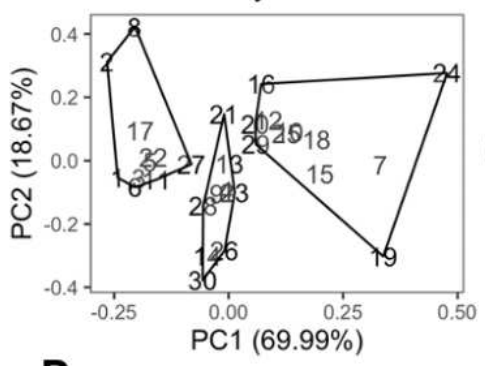

D CSR

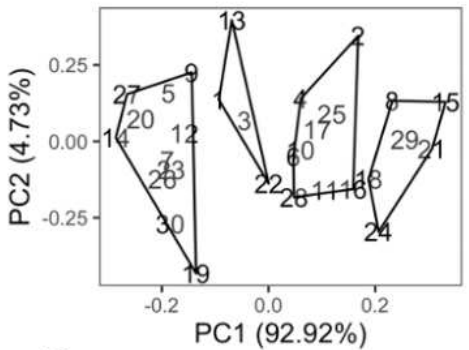

G LES

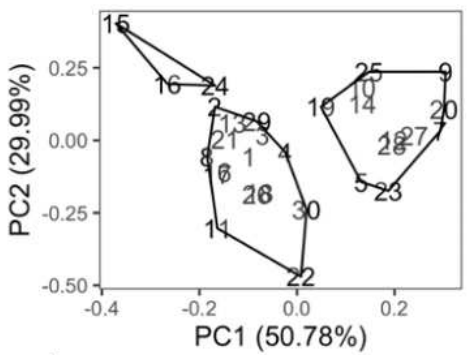

J All traits

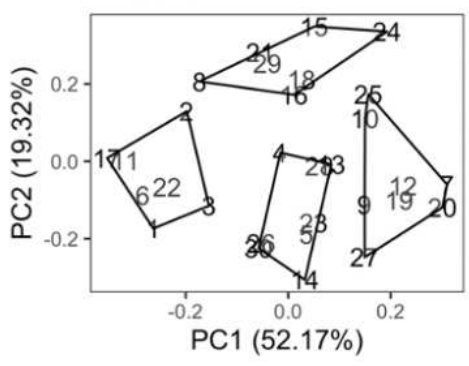

B Growth analysis

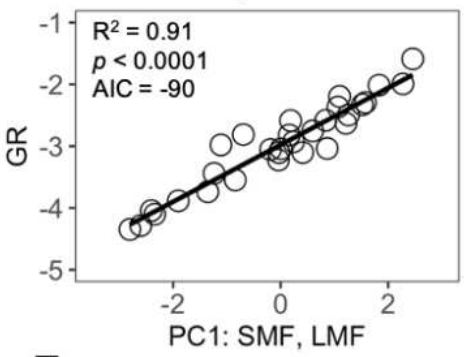

$E_{\text {CSR }}$

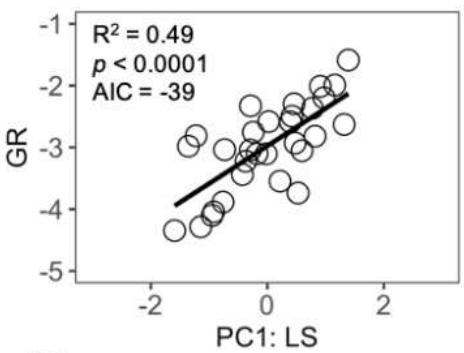

$\mathbf{H}_{\text {LES }}$

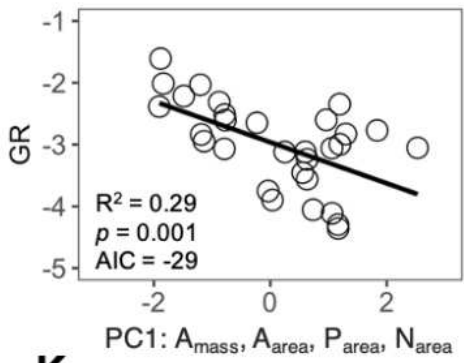

$\mathbf{K}_{\text {All traits }}$

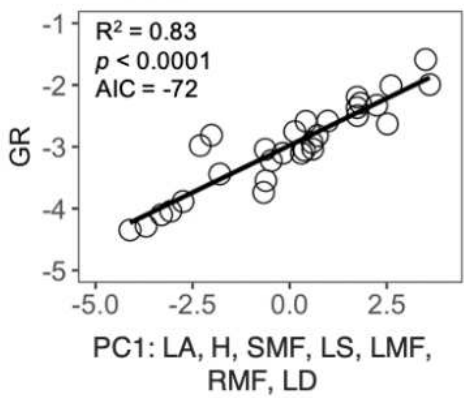

C Growth analysis

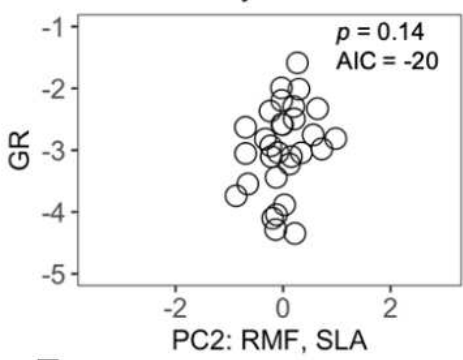

$F_{\text {CSR }}$

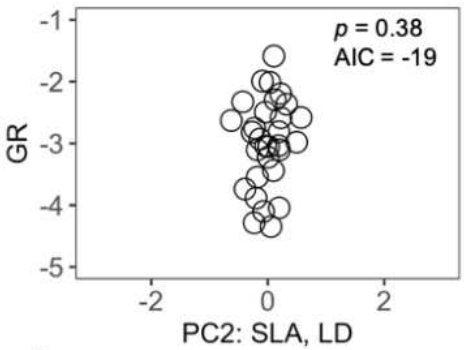

I LES

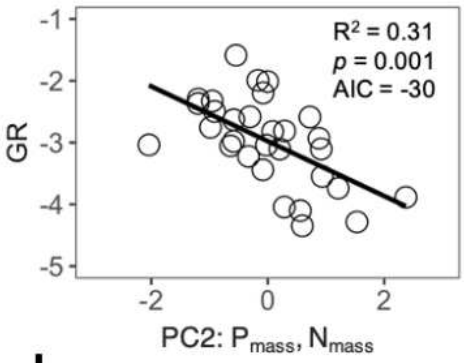

L All traits

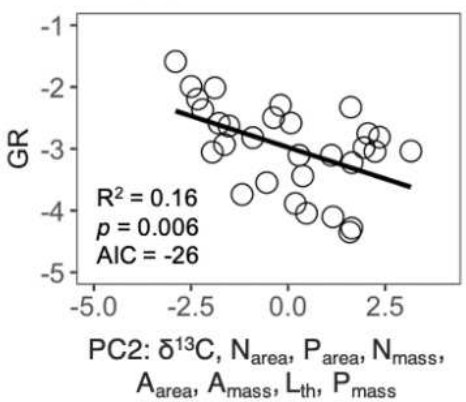

489

Figure 1. Biplots of principal components analyses (PCA) of 30 Asclepias species based on the defining traits of three classic ecological theories: (A) growth analysis, (B) CSR, (C) LES, and (D) 21 measured traits. Species groups are represented as convexes based on Ward's agglomerative clustering defined by trait similarity. (E-H) Correlations between growth rate and $\mathrm{PC} 1$, and (I-L) between growth rate and PC2. Variables with the highest eigenvector scores on PC1 and PC2 are presented from left to right for each axis. Data are species means, species names are in Table 3. 
498 Table 1. Traits and growth predictions of growth analysis, CSR triangle, and leaf economics 499 spectrum. '+' and '-' indicate positive and negative correlations, respectively.

\begin{tabular}{|c|l|c|}
\hline \multicolumn{1}{|c|}{ Strategy Scheme } & \multicolumn{1}{|c|}{ Traits } & Growth predictions \\
\hline \multirow{5}{*}{ Growth analysis } & Leaf mass fraction (LMF) & + \\
& Stem mass fraction (SMF) & + \\
& Root mass fraction (RMF) & - \\
& Specific leaf area (SLA) & + \\
\hline \multirow{3}{*}{ Competition-Stress tolerant- } & Average leaf size (LS) &,+- \\
Ruderal (CSR) triangle & Leaf dry matter content (LD) & - \\
& Specific leaf area (SLA) & + \\
\hline \multirow{5}{*}{ Leaf economics spectrum } & Photosynthetic rate (Amax) & + \\
& Respiration rate (Rd) & + \\
& Specific leaf area (SLA) & + \\
& Leaf nitrogen content (N) & + \\
& Leaf phosphorus content (P) & + \\
& Leaf lifespan & - \\
\hline
\end{tabular}


Table 2. Linear correlations between 21 plant traits and growth rate (GR) using generalized linear models (GLM) and phylogenetic least squares regression (PGLS) for 30 Asclepias species grown under common conditions. Sign indicates the direction of the significant correlation (positive, + or negative, -). Phylogenetic signal using Pagel's $\lambda$ is shown for each trait, with $P<$

5050.05 indicating significant phylogenetic signal.

\begin{tabular}{|c|c|c|c|c|c|c|c|}
\hline \multirow{3}{*}{ Trait } & \multicolumn{5}{|c|}{ Correlations with GR } & \multirow[t]{3}{*}{$\lambda$} & \multirow[t]{3}{*}{$\boldsymbol{P}$} \\
\hline & \multirow[b]{2}{*}{ Sign } & \multicolumn{2}{|c|}{ GLM } & \multicolumn{2}{|c|}{ PGLS } & & \\
\hline & & $R^{2}$ & $\boldsymbol{P}$ & $\boldsymbol{R}^{2}$ & $\boldsymbol{P}$ & & \\
\hline$\delta^{13} \mathrm{C}$ (carbon isotope ratio, \%o) & + & 0.35 & 0.001 & 0.36 & 0.0005 & 0.70 & 0.03 \\
\hline $\mathrm{H}$ (height, $\mathrm{cm}$ ) & + & 0.47 & $<0.0001$ & 0.36 & 0.0005 & 0.99 & $<0.001$ \\
\hline LA (leaf area total, $\mathrm{cm}^{2}$ ) & + & 0.56 & $<0.0001$ & 0.61 & $<0.0001$ & 0.18 & 0.37 \\
\hline \multicolumn{8}{|l|}{$\mathrm{R}_{\mathrm{d}}$ (leaf dark respiration rate) } \\
\hline per area $\left(\mathrm{R}_{\text {area}}, \mu \mathrm{mol} \mathrm{m} \mathrm{m}^{-2} \mathrm{~s}^{-1}\right)$ & n.s & 0.02 & 0.41 & 0.01 & 0.67 & $<0.001$ & 0.98 \\
\hline per mass $\left(R_{\text {mass }} \mu \mu_{\text {mol g }}{ }^{-1} \mathrm{~s}^{-1}\right)$ & n.s & 0.01 & 0.53 & 0.004 & 0.73 & 0.93 & 0.003 \\
\hline LD (leaf density, $\mathrm{mg} \mathrm{g}^{-1}$ ) & + & $\mathbf{0 . 1 0}$ & 0.05 & 0.17 & 0.02 & 0.64 & 0.05 \\
\hline LMF (leaf mass fraction, \%) & + & 0.14 & 0.04 & 0.01 & 0.64 & 0.92 & 0.01 \\
\hline \multicolumn{8}{|l|}{$\mathrm{N}$ (leaf nitrogen) } \\
\hline per area $\left(\mathrm{N}_{\text {area }}, \mathrm{gN} \mathrm{m}^{-2}\right)$ & - & 0.27 & 0.004 & 0.20 & 0.01 & 0.45 & 0.29 \\
\hline per mass $\left(\mathrm{N}_{\text {mass }}, \mathrm{gN} \mathrm{g}^{-1}\right)$ & - & 0.38 & 0.0003 & 0.50 & $<0.0001$ & 0.97 & 0.001 \\
\hline \multicolumn{8}{|l|}{$\mathrm{P}$ (leaf phosphorus) } \\
\hline per area $\left(\mathrm{P}_{\text {area }}, \mathrm{gP} \mathrm{m}^{-2}\right)$ & - & 0.51 & $<0.0001$ & 0.55 & $<0.0001$ & 0.06 & 0.72 \\
\hline per mass $\left(\mathrm{P}_{\text {mass }}, \mathrm{gP} \mathrm{g}^{-1}\right)$ & - & 0.26 & 0.004 & 0.19 & 0.02 & 0.03 & 0.88 \\
\hline LS (leaf size average, $\mathrm{cm}^{2}$ ) & + & 0.24 & 0.04 & 0.31 & 0.001 & $<0.001$ & 0.98 \\
\hline $\mathrm{L}_{\text {th }}$ (leaf thickness, mm) & n.s & 0.06 & 0.20 & 0.05 & 0.24 & 0.74 & $\mathbf{0 . 0 2}$ \\
\hline \multicolumn{8}{|l|}{ A (photosynthetic rate) } \\
\hline per area $\left(\mathrm{A}_{\text {area}}, \mu \mathrm{mol} \mathrm{m} \mathrm{m}^{-2} \mathrm{~s}^{-1}\right)$ & + & 0.23 & 0.008 & 0.32 & 0.001 & 0.49 & 0.97 \\
\hline per mass (Amass, $\left.\mu \mathrm{mol} \mathrm{g} \mathrm{g}^{-1} \mathrm{~s}^{-1}\right)$ & + & 0.03 & 0.34 & 0.19 & 0.01 & 0.70 & 0.20 \\
\hline Root clonality (\# of buds) & n.s & 0.01 & 0.73 & 0.03 & 0.34 & $<0.001$ & 0.98 \\
\hline RMF (root mass fraction, \%) & n.s & 0.08 & 0.13 & 0.01 & 0.67 & 0.50 & 0.17 \\
\hline SM (seed mass, g) & n.s & 0.01 & 0.63 & 0.04 & 0.30 & 0.99 & 0.001 \\
\hline SLA (specific leaf area, $\mathrm{cm}^{2} \mathrm{~g}^{-1}$ ) & n.s & 0.001 & 0.88 & 0.01 & 0.96 & $<0.001$ & 0.98 \\
\hline SRL (specific root length, $\mathrm{cm}^{2} \mathrm{mg}^{-1}$ ) & n.s & 0.05 & 0.25 & 0.06 & 0.19 & $<0.001$ & 0.98 \\
\hline SMF (stem mass fraction, $\%$ ) & + & 0.36 & 0.0004 & 0.17 & 0.02 & 0.95 & $<0.001$ \\
\hline
\end{tabular}


Table 3. Average growth rate (GR, $\left.\mathrm{g} \mathrm{d}^{-1}\right)$ and strategic classifications for 30 Asclepias species grown under common conditions. Species are in order by increasing GR. Growth analysis (GA) strategies are defined by species' predominant biomass fraction (root, stem, leaf); CSR by competitive (C), stress tolerant (S), ruderal (R), or their combination. Leaf economic spectrum

512 (LES) strategies are defined as resource acquisitive or conservative. Strategies identified by

513 comparing all 21 measured traits are defined by relative plant size (small, medium, large) and

514 predominant biomass fraction (root, stem, leaf).

\begin{tabular}{|c|c|c|c|c|c|c|}
\hline & & & \multicolumn{4}{|c|}{ Strategy } \\
\hline \multicolumn{2}{|r|}{ Species } & GR & GA & CSR & LES & All traits \\
\hline 17 & A. longifolia & 0.013 & root & SR & acq/cons & small, root \\
\hline 11 & A. hirtella & 0.014 & root & SR & acq/cons & small, root \\
\hline 6 & A. cryptoceras & 0.017 & root & SR & $\mathrm{acq} / \mathrm{cons}$ & small, root \\
\hline 1 & A. amplexicaulis & 0.018 & root & CSR & $\mathrm{acq} / \mathrm{cons}$ & small, root \\
\hline 22 & A. solanoana & 0.021 & root & CSR & $\mathrm{acq} / \mathrm{cons}$ & small, root \\
\hline 30 & A. viridis & 0.024 & leaf & $\mathrm{CR}$ & acq/cons & medium, leaf \\
\hline 26 & A. sullivantii & 0.029 & leaf & $\mathrm{CR}$ & acq/cons & medium, leaf \\
\hline 3 & A. asperula & 0.032 & root & CSR & acq/cons & small, root \\
\hline 29 & A. verticillata & 0.040 & stem & $\mathrm{S}$ & acq/cons & medium, stem \\
\hline 4 & A. brachystephana & 0.045 & leaf & SR & $\mathrm{acq} / \mathrm{cons}$ & medium, leaf \\
\hline 18 & A. mexicana & 0.045 & stem & $\mathrm{S}$ & $\mathrm{acq} / \mathrm{cons}$ & medium, stem \\
\hline 14 & A. latifolia & 0.047 & leaf & $\mathrm{CR}$ & conservative & medium, leaf \\
\hline 21 & A. pumila & 0.048 & leaf & $\mathrm{S}$ & acquisitive & medium, stem \\
\hline 15 & A. linaria & 0.048 & leaf & $\mathrm{S}$ & acq/cons & medium, stem \\
\hline 2 & A. arenaria & 0.051 & root & SR & $\mathrm{acq} / \mathrm{cons}$ & small, root \\
\hline 23 & A. speciosa & 0.053 & leaf & $\mathrm{CR}$ & conservative & medium, leaf \\
\hline 28 & A. tuberosa & 0.060 & leaf & $\mathrm{S}$ & $\mathrm{acq} / \mathrm{cons}$ & medium, leaf \\
\hline 8 & A. engelmanniana & 0.060 & root & SR & conservative & medium, stem \\
\hline 16 & A. linearis & 0.064 & stem & SR & acquisitive & medium, stem \\
\hline 19 & A. perennis & 0.072 & stem & $\mathrm{CR}$ & conservative & large, leaf \& stem \\
\hline 5 & A. californica & 0.075 & leaf & $\mathrm{CR}$ & conservative & medium, leaf \\
\hline 13 & A. labriformis & 0.076 & leaf & CSR & acq/cons & medium, leaf \\
\hline 10 & A. fascicularia & 0.082 & stem & SR & conservative & large, leaf \& stem \\
\hline 9 & A. eriocarpa & 0.094 & leaf & $\mathrm{CR}$ & conservative & large, leaf \& stem \\
\hline 24 & A. subulata & 0.098 & stem & $\mathrm{S}$ & acquisitive & medium, stem \\
\hline 25 & A. subverticillata & 0.101 & stem & SR & conservative & large, leaf \& stem \\
\hline 27 & A. syriaca & 0.111 & leaf & $\mathrm{CR}$ & conservative & large, leaf \& stem \\
\hline 12 & A. incarnata ssp. incarnata & 0.134 & stem & $\mathrm{CR}$ & conservative & large, leaf \& stem \\
\hline 7 & A. curassavica & 0.136 & stem & $\mathrm{CR}$ & conservative & large, leaf \& stem \\
\hline 20 & A. incarnata ssp. pulchra & 0.205 & stem & $\mathrm{CR}$ & conservative & large, leaf \& stem \\
\hline
\end{tabular}




\section{Supplementary Files}

This is a list of supplementary files associated with this preprint. Click to download.

- GoudetalGrowth2021SI.pdf 